\title{
Matrix metalloproteinase-12 (MMP-12) SNP affects MMP activity, lung macrophage infiltration and protects against emphysema in COPD
}

\author{
Imran Haq, ${ }^{1}{ }^{2}$ Gillian E Lowrey, ${ }^{1}$ Noor Kalsheker, ${ }^{2}$ Simon R Johnson ${ }^{1}$
}

\begin{abstract}
- Additional materials are published online only. To view these files please visit the journal online (http://thorax.bmj. com)

${ }^{1}$ Division of Therapeutics and Molecular Medicine, NIHR Respiratory Biomedical Research Unit, Queens Medical Centre, University of Nottingham, Nottingham, UK ${ }^{2}$ Division of Clinical Chemistry. Queen's Medical Centre, University of Nottingham, Nottingham, UK
\end{abstract}

\section{Correspondence to}

Simon Johnson, Division of Therapeutics and Molecular Medicine and NIHR Respiratory Biomedical Research Unit, Queens Medical Centre, University of Nottingham, D Floor, South Block, Queen's Medical Centre, Nottingham NG7 2UH, UK; simon.johnson@ nottingham.ac.uk

NK and SRJ are joint senior authors.

Received 20 January 2011 Accepted 9 June 2011 Published Online First 5 July 2011

\begin{abstract}
Background Recent genetic and animal studies have implicated matrix metalloproteinase-12 (MMP-12) in the pathogenesis of chronic obstructive pulmonary disease (COPD). It has previously been shown that individuals homozygous for the A/A allele of rs652438 in MMP-12 are over-represented among patients with severe COPD $(n=1517)$. A study was undertaken to examine the functional basis of these findings.
\end{abstract}

Methods rs652438 A and $G$ variants were generated by site-directed mutagenesis and transfected into COS7 cells where they were expressed. Casein zymography and a specific FRET activity assay were used to compare MMP-12 activity between alleles. Cell migration was examined using a transwell assay. Patients from two COPD cohorts were genotyped for rs652438 and associated with inflammatory cell number in bronchoalveolar lavage fluid $(n=10)$ and induced sputum $(n=262)$; the emphysema score $(n=1428)$ was assessed by CT scanning.

Results Mean MMP activity was 2.95-fold higher by zymography $(p=0.0049)$ and 3.45 -fold higher by FRET assay ( $p=0.0001$ ) for the $A$ allele than the $G$ allele. Mean migration of COS7 cells expressing the A allele was 2.31-fold greater than for those expressing the $G$ allele $(p=0.0001)$. Macrophage numbers were greater in bronchoalveolar lavage fluid (1.28-fold increase, $p=0.033)$ and induced sputum (1.58-fold increase, $p=0.083$ ) of $A / A$ individuals compared with $A / G$ heterozygotes. The presence of the $A$ allele was dose-dependently associated with increased emphysema $(p=0.016)$.

Conclusions The rs652438 SNP alters MMP-12 activity with the A allele being more active, which is associated with increased macrophage infiltration and emphysema in the lungs of patients with COPD. These findings further implicate MMP-12 and this SNP in COPD.

\section{INTRODUCTION}

It is widely accepted that alveolar destruction in chronic obstructive pulmonary disease (COPD) is the result of an imbalance of proteolytic enzymes and their inhibitors based on the observation that severe $\alpha_{1}$-antitrypsin deficiency predisposes cigarette smokers to the development of pulmonary emphysema. This is thought to be due to uninhibited neutrophil elastase activity degrading elastin, a major component of lung connective tissue. ${ }^{1}$

A number of other proteolytic enzymes are also capable of degrading elastin, including the matrix metalloproteinases (MMPs). These proteases can degrade all the major protein components of the extracellular matrix (ECM). Evidence from genetic

\section{Key messages}

What is the key question?

- How does a single nucleotide polymorphism in the haemopexin-like domain of MMP-12 protect patients against severe COPD?

What is the bottom line?

- The protective variant of MMP-12 reduces metalloproteinase activity and cell migration in vitro and is associated with lower lung macrophage numbers and emphysema in patients with COPD.

\section{Why read on?}

- By investigating how this genetic variant in MMP-12 protects against severe COPD, this study addresses one of the factors contributing to the idea of the 'susceptible smoker' in COPD.

studies, animal models and human disease suggest that MMP-12, the major metalloelastase derived from alveolar macrophages, plays a role in lung destruction in COPD. ${ }^{2-6}$ Recently, a large multiple cohort association study linked variation in MMP12 with both lung function in smokers and the risk of developing $\mathrm{COPD}^{2}$ while, in animal models of COPD, deletion of MMP-12 in mice exposed to cigarette smoke protects these animals from emphysema. ${ }^{3}$ In patients with COPD elevated levels of MMP-12 are present in the airways ${ }^{4} 5$ and increased numbers of MMP-12 expressing macrophages. ${ }^{6}$

We recently performed a case-control genetic association study into MMPs 1, 9 and 12. This identified haplotypes in MMP-12 of two single nucleotide polymorphisms (SNPs), rs652438 and rs2276109, which were associated with severe and very severe COPD (GOLD stages III and IV). In the study by Hunninghake et al ${ }^{2}$ rs652438 was not associated, possibly due to different phenotypes tested. This suggests that these SNPs are modifiers of disease severity, and supports the idea that MMP-12 is a mediator of lung damage in COPD. ${ }^{7}$ In our previous study about $18 \%$ of Europeans were found to have at least one protective allele at these SNP positions, giving a significantly reduced risk of severe disease development. ${ }^{7}$

The SNPs at rs2276109 and rs652438 are located in the promoter region of the gene and haemopexin domain of the enzyme, respectively, and could directly influence MMP-12 activity through altered 
expression or substrate binding. The former has been demonstrated for rs2276109, where the A allele in the MMP-12 promoter results in increased MMP-12 transcription. ${ }^{8}$ The rs652438 A/G SNP causes a missense change of asparagine to serine at amino acid position 357, located in exon 8, which encodes the haemopexin domain. The haemopexin domain of MMP-12 is involved in substrate specificity and may interact with the enzyme's catalytic domain. ${ }^{9}$ We hypothesised that this SNP could alter the function of MMP-12, thereby altering the pathological processes in COPD.

\section{MATERIAL AND METHODS}

Additional data on methods are provided in the online supplement.

\section{Patient cohorts}

Two cohorts were used in this study, a local Nottingham cohort of patients with $\mathrm{COPD}^{10}$ and ECLIPSE (Evaluation of COPD Longitudinally to Identify Predictive Surrogate End-points). ${ }^{11}$

\section{Nottingham cohort}

We recruited smokers with COPD defined by GOLD criteria. Patients were aged 40-80 years, had a smoking history of at least 10 pack-years and had not required antibiotics or oral steroids for the previous 6 weeks. Patients with $\alpha_{1}$-antitrypsin deficiency, radiological evidence of interstitial lung disease, previous thoracic surgery or taking inhaled corticosteroids were excluded. Pre- and post-bronchodilator spirometry was performed according to American Thoracic Society guidelines and breathlessness was recorded using the Medical Research Council (MRC) dyspnoea score. Those with reversibility $>10 \%$ of baseline forced expiratory volume in $1 \mathrm{~s}\left(\mathrm{FEV}_{1}\right)$ after inhaling $400 \mu \mathrm{g}$ salbutamol were excluded. Subjects with $\mathrm{FEV}_{1} /$ forced vital capacity (FVC) of $<70 \%$ were classified as having COPD.

\section{ECLIPSE cohort}

Patients with COPD aged 40-75 years with baseline postbronchodilator $\mathrm{FEV}_{1}<80 \%$ of the predicted value, baseline postbronchodilator $\mathrm{FEV}_{1} / \mathrm{FVC}$ of $<0.7$ and a smoking history of $>10$ pack-years were enrolled. Exclusion criteria were related to diagnosis (known respiratory disorders other than COPD and severe $\alpha_{1}$-antitrypsin deficiency), prior medical history (known history of significant inflammatory disease other than COPD), a COPD exacerbation within 4 weeks of enrolment, having undergone lung surgery, recent diagnosis of cancer, having received a blood transfusion in the 4 weeks before the start of the study, inability to walk, taking part in a blinded drug study, treatment with oral corticosteroids at inclusion and participation in studies with radiation exposure.

Information on the cell percentage composition of bronchoalveolar lavage fluid (BAL) was provided for the Nottingham cohort. Absolute cell number and emphysema scores were provided for ECLIPSE, providing some replication for the cell number measure described by the Nottingham cohort. Emphysema scores were obtained using CT scanning performed at full suspended inspiration. A multislice CT scanner was used, taking contiguous images at 1 or $1.25 \mathrm{~mm}$ thickness. Scanning was set at $120 \mathrm{kVp}$ and $40 \mathrm{mAs}$. A low spatial frequency reconstruction algorithm was used for analysis (GE-standard, Siemens Healthcare, Erlangen, Germany). Genotypes of rs652438 were determined in the Nottingham cohort by direct sequencing. ECLIPSE genotypes were provided using methods described elsewhere. ${ }^{11}$

\section{Vector creation}

MMP-12 (pCMV-XL4 vector) was obtained from Origene (Rockville, USA). The rs652438 SNP G allele was introduced via the GeneEditor kit (Promega, Southampton, UK) according to the manufacturer's instructions using mutagenic primer 5'-TAATTAGCAGTTTAAGACC-3'. After DNA preparation (Qiagen, Crawley, UK), inserts were sequenced and aligned using ClustalW (http://www.ebi.ac.uk/Tools/clustalw2/index.html) to confirm inserts were identical apart from the SNP. The vector without an insert was used as null control in all experiments.

\section{Cell culture}

COS7 cells were grown in DMEM using 10\% fetal bovine serum at $37^{\circ} \mathrm{C}$ and $5 \% \mathrm{CO}_{2}$. Vectors were transiently transfected using Fugene 6 (Roche, Mannheim, Germany). After $24 \mathrm{~h}$ incubation, cells were serum starved, supernatants collected after $24 \mathrm{~h}$ and stored at $-80^{\circ} \mathrm{C}$.

\section{Analysis of enzymatic activity}

Casein zymography was used to perform analysis of activity as detailed elsewhere. ${ }^{12}$ Changes to the protocol were $12 \%$ casein gels (Invitrogen, Paisley, UK) and quantification against $10 \mathrm{ng}$ recombinant human MMP-12 (R\&D Systems, Minneapolis, USA). Images were scanned and analysed using Image J V.1.40 (Wayne Rasband, NIH, USA). Bands were normalised against standard and presented as a ratio.

MMP-12 activity was also measured using the Sensolyte 520 MMP-12 assay kit (Anaspec, San Jose, California, USA) according to the manufacturer's instructions. The assay was run on a Flexstation with Softmax Pro software (Molecular Devices, Sunnyvale, California, USA) at excitation/emission wavelengths of 490/520 nm respectively. Recombinant human MMP-12 (10 ng) was the reference standard. Substrate-only samples were included for background subtraction purposes. All measurements were performed in triplicate and the experiments were repeated three times.

\section{Western blot}

Supernatants and human MMP-12 standard (10 ng) were resolved using $10 \%$ SDS page gels followed by blotting onto Hybond-P membrane (GE Healthcare, Buckinghamshire, UK) and probed with IgG anti-human MMP-12 catalytic antibody (R\&D Systems, Minneapolis, USA). HRP-conjugated goat antimouse IgG (Sigma Aldrich, Gillingham, UK) was used. Visualisation was performed using the ECL western blotting detection kit (GE Healthcare).

\section{Cell viability assays}

Total cell counts and cell viability measurements were performed using a haemocytometer and MTT assay as described elsewhere. ${ }^{12} 13$ Quantification of total protein levels in supernatants was performed using a Bradford based method ${ }^{14}$ (BioRad Protein Assay, Berkley, California, USA).

\section{Real-time PCR}

Quantification of MMP-12 mRNA was performed as described elsewhere. ${ }^{12} \beta$-actin was used for normalisation. Primer sequences are as follows: MMP-12 forward: 5'-CATTCAGGAGGCACCAACTTGTTC-3', reverse: 5'-CCTTTGGATCACTAGAATGGCC-3'; $\beta$-actin forward: $5^{\prime}$-GGATGCAGAAGGAGATTACTG-3', reverse: 5 '-CGATCCACACAGAGTACTTG-3'

\section{Minigene analysis of splicing}

Exonic splice silencing was analysed using the Minigene technique. Details of the method used are provided in the online supplement. 
Table 1 Basic characteristics of COPD cohorts

\begin{tabular}{llll}
\hline & Nottingham & $\begin{array}{l}\text { ECLIPSE: } \\
\text { cell numbers }\end{array}$ & $\begin{array}{l}\text { ECLIPSE: } \\
\text { emphysema score }\end{array}$ \\
\hline Male (\%) & 80.0 & 63.2 & 67.0 \\
Age (years) & $59.4 \pm 3.1$ & $64.0 \pm 0.4$ & $63.6 \pm 0.2$ \\
Smoking (pack-years) & $60.6 \pm 7.1$ & $50.9 \pm 1.6$ & $50.4 \pm 0.7$ \\
FEV $_{1}$ (\% predicted) & $59.0 \pm 7.3$ & $49.2 \pm 0.9$ & $47.7 \pm 0.4$ \\
FEV $_{1} /$ FVC (\%) & $55.45 \pm 4.0$ & $43.81 \pm 0.7$ & $44.7 \pm 0.3$ \\
$\mathrm{~N}$ & 10 & 232 & 1427 \\
\hline
\end{tabular}

COPD, chronic obstructive pulmonary disease; ECLIPSE, Evaluation of COPD Longitudinally to Identify Predictive Surrogate End-points; FEV 1 , forced expiratory volume in $1 \mathrm{~s}$; FVC, forced vital capacity.

\section{Migration assay}

The transwell migration assay was performed as previously described. ${ }^{12}$ Changes to the protocol were use of collagen IV $(0.1 \mathrm{mg} / \mathrm{ml}$ in serum-free medium) coating and $10 \%$ serum as a chemotactic stimulus. Migration was measured at $8 \mathrm{~h}$ in three replicates in three independent experiments.

\section{Statistical analysis}

Data sets were tested for normality using the D'Agostino and Pearson test and analysed using t tests (Prism 4, Graphpad Software, La Jolla, USA). Linear regression of SensoLyte 520 kinetic assay was also performed in Prism 4. Cohort characteristics were investigated using the descriptive statistics function in SPSS V.14 (SPSS Inc). Analysis of the ECLIPSE cohort was performed using a linear regression model adjusted for confounding factors in SPSS V.14. For all tests, $p$ values of $<0.05$ were considered statistically significant.

\section{RESULTS}

\section{Study population characteristics}

The characteristics of the populations used in this study are detailed in table 1 .

\section{Analysis of enzymatic activity}

MMP-12 activity was examined using casein zymography of COS7 supernatants from cells expressing the A and G constructs. COS7 cells expressing the null vector had no detectable caseinolytic activity (limit of detection $1 \mathrm{ng}$ per lane). In supernatants from COS7 cells expressing the MMP-12 constructs, caseinolytic activity was present at $54 \mathrm{kDa}$ and $45 \mathrm{kDa}$, corresponding to the pro- and intermediate-active forms of MMP-12 (figure 1). Using the t test and comparison of the means, densitometry of caseinolytic bands showed that the activity of the A allele was 2.95-fold ( $p=0.0049)$ higher than that of the $G$ allele.

We next used the Sensolyte 520 MMP-12 ELISA. Using the $t$ test and comparison of the means, the A allele of rs652438 was 3.42 -fold ( $p=0.0001)$ more active than the $\mathrm{G}$ allele (figure 1$)$. The Sensolyte assay was also used to provide a kinetic read of MMP-12 activity (figure 2). Using the linear regression function in Prism, the slope of substrate cleavage against time for the A allele was 1.6 $\mathrm{CI} \pm 0.106$ relative fluorescence units/min (RFU) ( $p=0.0001$ vs empty vector) and the $G$ allele slope was $0.12 \mathrm{CI} \pm 0.096 \mathrm{RFU}$ ( $p=0.22$ vs empty vector). The MMP-12 activity of the $G$ allele was not different $(p=0.2153)$ from the null vector baseline.

\section{Effect of SNP on cell viability and MMP-12 expression}

Examination of MMP-12 constructs was performed to determine whether the SNP affects MMP-12 mRNA expression, cell number or viability. Real-time PCR and Minigene determined

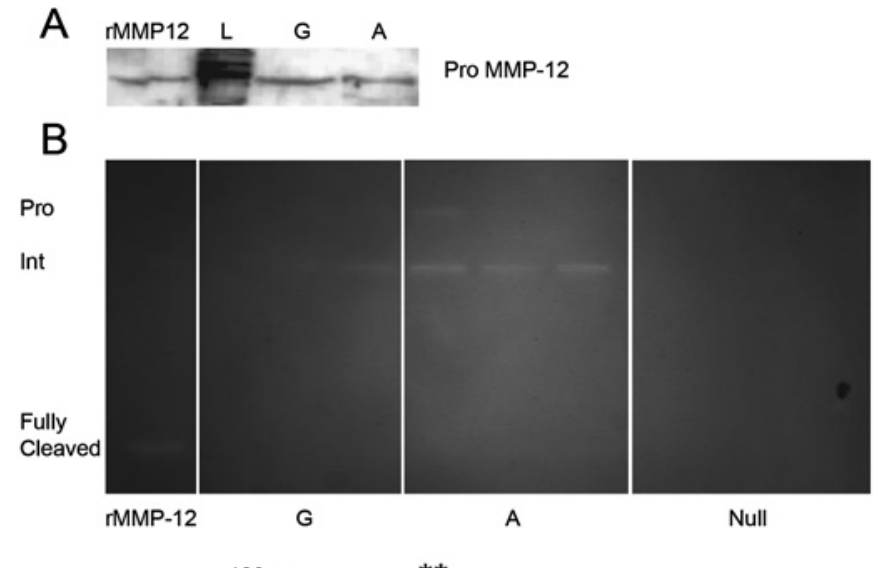

Figure 1 (A) Western blot of rs652438 A and G paired transfections. Expression levels of matrix metalloproteinase-12 (MMP-12) were found to be equal between the transfection of the different constructs. (B) Representative casein zymogram showing expression of the pro and intermediate forms of MMP-12 from vector-transfected cell supernatants. Below, densitometry of three independent experiments. The A allele has mean \pm SEM band intensity of $77.11 \pm 14.26 \%$ compared with the $10 \mathrm{ng}$ MMP-12 standard while the is $G$ allele has a band intensity of $26.11 \pm 6.39 \%$. The $A$ allele activity is 2.95 -fold higher than the $G$ allele $(p=0.0049)$. (C) Sensolyte 520 MMP-12 assay endpoint read. The A allele has mean \pm SEM activity of $48.30 \pm 3.44 \%$ compared with the $10 \mathrm{ng}$ MMP-12 standard while the is $\mathrm{G}$ allele has $14.13 \pm 1.30 \%$ activity. The A allele activity is 3.42 -fold higher than the $G$ allele $(p=0.0001 ; n=3)$.

that the rs652438 polymorphism did not influence mRNA levels (figure 3). Using the t test, no significant differences were seen in cell number, supernatant total protein or viability (figure 4).

\section{Cell migration}

Transwells were coated with collagen IV, a non-fibrillar collagen expressed in basement membranes which can be degraded by MMP-12. ${ }^{15}$ Using t tests and mean values as a comparator, both MMP-12 allele transfected cells had increased migration compared to cells containing the null vector. Further to this, A allele containing cells had significantly increased migration (2.31-fold increase, $p=0.0001)$ when compared to those transfected with the $\mathrm{G}$ allele (figure 5). 


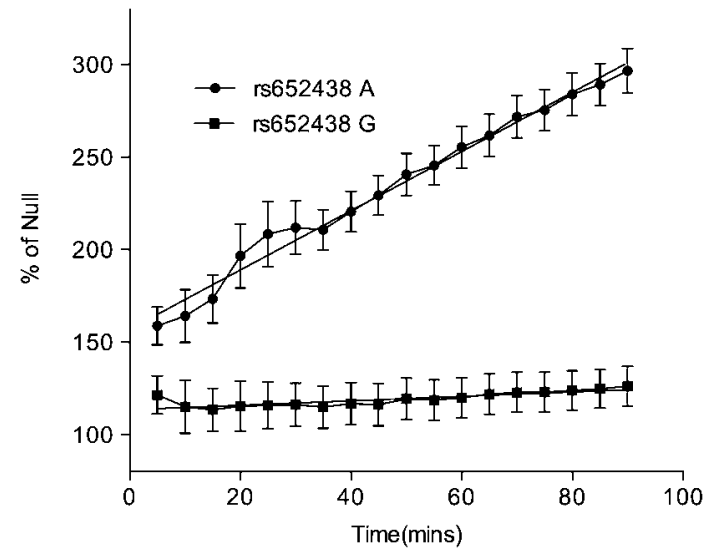

Figure 2 Sensolyte 520 MMP-12 assay kinetic read. The A allele of rs652438 is significantly different from the null allele baseline slope $(1.6000 \pm 0.1062, p=0.0001)$ while the $G$ allele is not significantly different from the baseline slope $(0.1201 \pm 0.0963, p=0.2153 ; n=3)$.

We investigated whether this effect on migration was relevant in patients with COPD. We examined data from the Nottingham cohort using BAL fluid samples in which the percentage composition values of inflammatory cells were available. Patients were genotyped for rs652438 using direct sequencing. Using a t test and comparing the means, we found a 1.28-fold $(n=10, p=0.033)$ increase in the percentage of BAL fluid macrophages for A/A homozygotes compared with A/G genotypes (figure 6). There has also been evidence that MMP-12 is involved in neutrophil recruitment to the airways ${ }^{16}$ but in our study we did not find any significant differences as determined by $t$ test. A/A genotypes had $4.820 \pm 2.239 \%$ composition of neutrophils while A/G genotypes had $21.040 \pm 9.058 \%$ composition $(p=0.0602)$.

In order to provide some replication, information from the ECLIPSE cohort was obtained regarding absolute inflammatory cell numbers according to genotype. As the rs652438 SNP is not present on the Illumina 550 genome-wide genotyping platform used in ECLIPSE, we studied the rs651159 intronic SNP which is in full linkage disequilibrium $\left(r^{2}=1\right)$ with rs652438 in HapMap CEU, EGP CEPH and AFD EUR submitter populations to the HapMap. ${ }^{17}$ Analysis of rs651159 allows $100 \%$ inference of rs652438 genotypes.

The focus of the analysis was the association of inferred rs652438 genotypes with absolute cell numbers of macrophages and neutrophils in induced sputum. In this large cohort, BAL fluid samples were not routinely taken and macrophage numbers were examined in induced sputum. Further to this, a comparable cell percentage phenotype in the induced sputum was not available. In the 232 patients for whom genotype and induced sputum data were available, there were $205 \mathrm{~A} / \mathrm{A}$ homozygotes, 27 A/G heterozygotes but no G/G homozygotes.
A

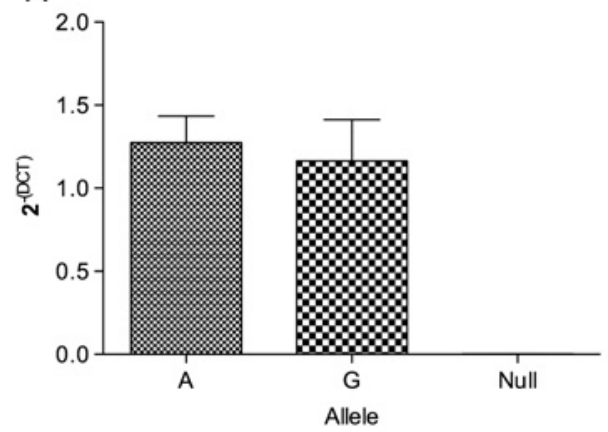

B

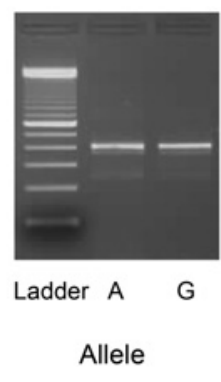

Figure 3 (A) Real-time PCR demonstrating robust and equal expression of matrix metalloproteinase-12 (MMP-12) constructs $(n=3)$. No endogenous MMP-12 was detected. (B) Real-time PCR of exon trap vector with MMP-12 exon 8 inserted. The $160 \mathrm{bp}$ exon 8 is transcribed along with the vector-specific exons, giving a 417 bp band. Because both bands are $417 \mathrm{bp}$ in size and of equal intensity, exonic silencing does not occur when the $G$ allele is present. If silencing had occurred, a $257 \mathrm{bp}$ band would be formed with only the vector-specific exons transcribed.

Absolute macrophage number in induced sputum was the dependent variable while genotype is the independent variable of interest. The final linear regression model was adjusted for smoking history, age, sex, pack-years and GOLD stage. Comparing the means showed a 1.58-fold increase in macrophage numbers in induced sputum samples of $\mathrm{A} / \mathrm{A}$ homozygotes compared with $\mathrm{A} / \mathrm{G}$ heterozygotes (figure 7), which did not reach statistical significance $\left(p=0.083\right.$, adjusted $\left.r^{2}=0.369\right)$ using a linear regression test.

The absolute neutrophil number was also investigated using linear regression adjusted for smoking history, age, sex, packyears and GOLD stage No significant differences $(p=0.306$, adjusted $\mathrm{r}^{2}=493$ ) were found between the $\mathrm{A} / \mathrm{A}$ group (mean $\pm \mathrm{SE}$ $2.48 \times 10^{6} \pm 0.25 \times 10^{6}$ cells per count) and the $A / G$ group (mean \pm SE $3.25 \times 10^{6} \pm 0.71 \times 10^{6}$ cells per count).

\section{Emphysema scores}

As in the previous investigation, the rs651159 SNP was used to infer the genotypes of rs652438 in ECLIPSE. There were $1282 \mathrm{~A} / \mathrm{A}$ homozygotic individuals, $141 \mathrm{~A} / \mathrm{G}$ heterozygotes and four $\mathrm{G} / \mathrm{G}$ minor homozygotes. The emphysema score determined by CT scanning was used as a quantitative trait in association analysis against genotype. The final linear regression model was adjusted for smoking history, age, sex, pack-years and GOLD stage. There was a dose-dependent effect on emphysema with each $G$ allele $\left(p=0.016\right.$, adjusted $r^{2}=0.245$ ), with a reduction in low attenuation areas between the $\mathrm{A} / \mathrm{A}$ major and $\mathrm{G} / \mathrm{G}$ minor homozygotes. Comparison of the means of the $A / A$ and $G / G$ homozygotes showed a 1.91-fold increase in the emphysema score (figure 8).

Figure 4 (A) Total cell count and (B) Bradford assay showing no significant differences in total cell number or total secreted protein levels in $A$ and $G$ alleles of matrix metalloproteinase-12 (MMP-12) rs652438 vector-transfected COS7 cells. (C) MTT assay indicating no significant differences in viable cells ( $n=3$ for all experiments).
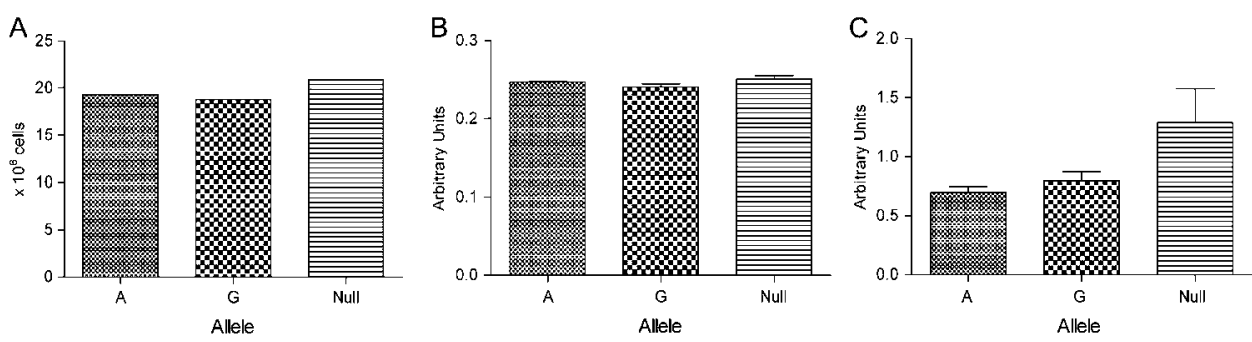
Figure 5 Chemotactic transwell migration assay $(n=3)$ showing a 2.31 fold $(p=0.0001)$ increase in migration for A allele transfected rs652438 COS7 cells compared with the $\mathrm{G}$ allele. Baseline used is the migration of null (N) vector-transfected COS7 cells. Cell migration photographs are representative of three independent experiments.

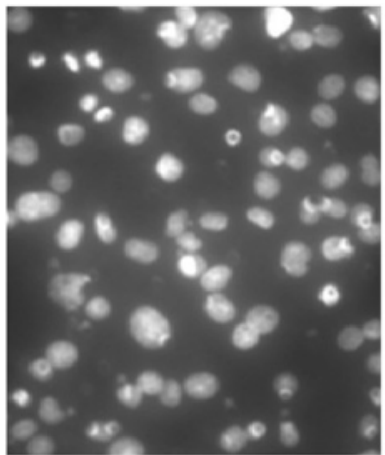

A

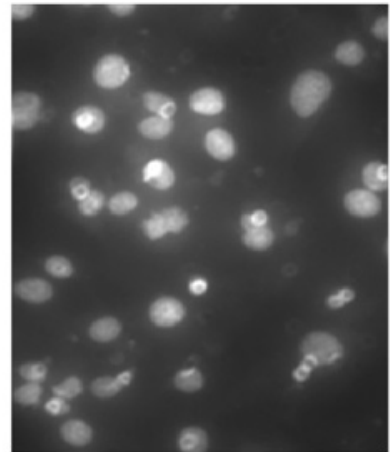

G

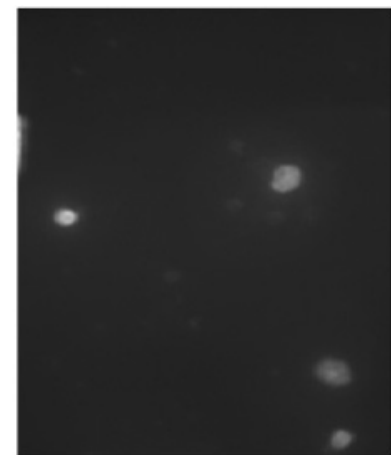

$\mathrm{N}$

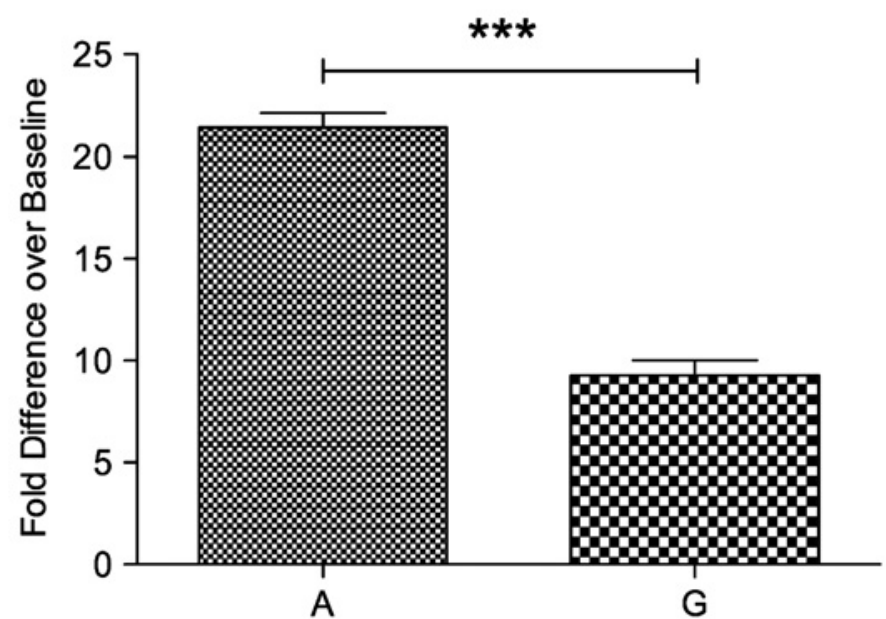

Allele

\section{DISCUSSION}

This study demonstrates that an exonic SNP alters the activity of MMP-12. Our findings provide evidence for the role of the rs652438 SNP in macrophage migration and emphysema severity, with the A allele over-represented in these cases. Consistent with these findings, we found that the A allele significantly increased the activity of MMP-12.

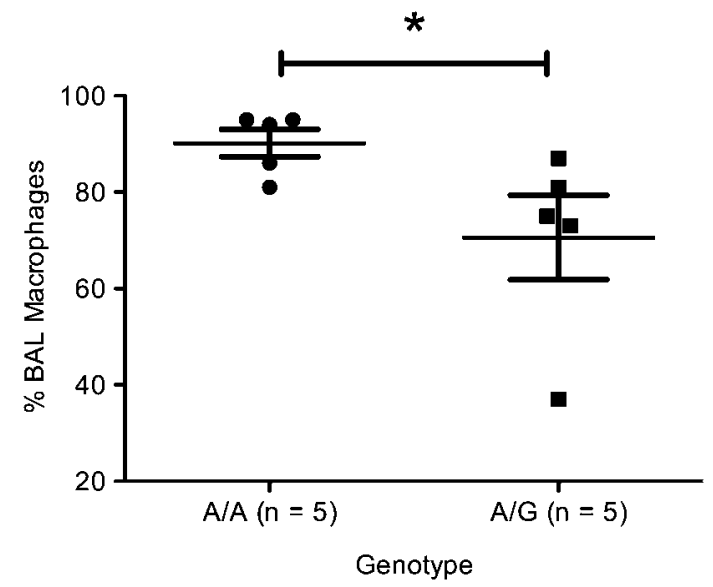

Figure 6 Percentage of macrophages in bronchoalveolar lavage (BAL) fluid from the Nottingham cohort. Individuals with $A / A$ genotype had mean \pm SEM $90.20 \pm 2.85 \%$ macrophages while $A / G$ individuals had $70.60 \pm 8.75 \%$ macrophages, a 1.18 -fold increase $(p=0.033)$.
The MMP-12 protein exists in three major forms: an inactive $54 \mathrm{kDa}$ form which has the propeptide domain cleaved to create the $45 \mathrm{kDa}$ active form, and this active $45 \mathrm{kDa}$ form is also processed to form a $22 \mathrm{kDa}$ protein with the haemopexin domain removed. Although processed to the $22 \mathrm{kDa}$ form, we do not think that this will have affected our in vitro findings as, in common with a previous study, we found that, in cell supernatants, the $45 \mathrm{kDa}$ form is stable under storage at $-80^{\circ} \mathrm{C}$ and for the duration of the assays. ${ }^{18}$ However, the purified recombinant MMP-12 readily underwent autocatalytic

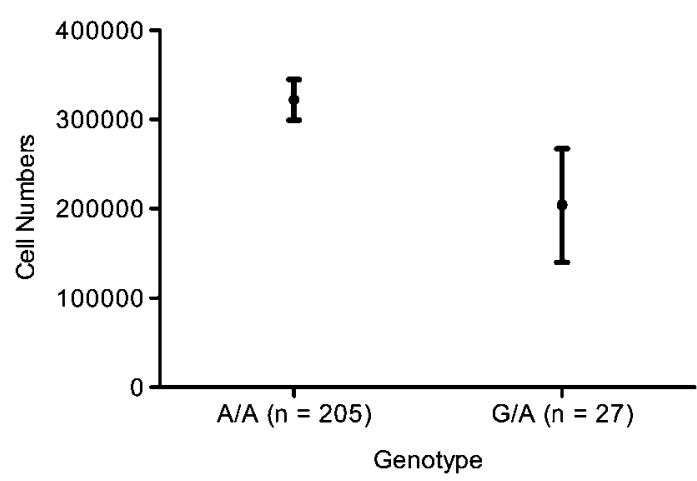

Figure 7 Absolute macrophage number in induced sputum by genotype in the ECLIPSE cohort. A/A homozygotic individuals had a mean $\pm S E$ of $3.22 \pm 0.23 \times 10^{5}$ cells per count compared with $2.04 \pm 0.64 \times 10^{5}$ cells per count for $\mathrm{A} / \mathrm{G}$ heterozygotes, a 1.58 -fold increase in cell number $\left(p=0.083\right.$, adjusted $\left.r^{2}=0.369\right)$. 


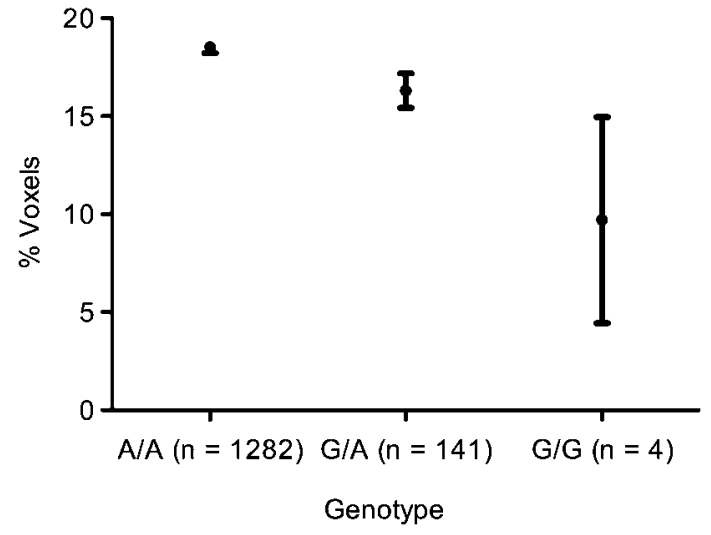

Figure 8 Emphysema score by genotype. The mean $\pm S E$ values for the different genotypes were: $A / A, 18.52 \pm 0.29 ; G / A, 16.31 \pm 0.89 ; G / G$, $9.70 \pm 5.26$. The increase between the major and minor homozygotes in emphysema scores is 1.91 -fold ( $p=0.016$, adjusted $\left.r^{2}=0.245 ; n=1427\right)$.

processing, suggesting the presence of stabilising factors in cell culture supernatants.

The haemopexin domain in MMP-12 has been hypothesised to have a flexible interaction with the catalytic domain compared with other MMPs, suggesting a role for the haemopexin domain in modulating enzymatic activity. ${ }^{19}$ The rs652438 SNP causes a neutral-polar mutation of arginine to serine, which suggests that the effect of the SNP seen in our study is through stabilisation of protein flexibility rather than a direct conformational change.

An interesting finding was the investigation of SNP alleles with regard to macrophage migration. The transwell migration assay set up to model macrophage invasion in the airways found significantly increased migration for the A allele. Furthermore, this finding was replicated in humans with statistically significant differences between individuals with $\mathrm{A} / \mathrm{A}$ and $\mathrm{A} / \mathrm{G}$ genotypes in the number/proportion of macrophages found in BAL fluid and induced sputum. However, it must be noted that grouped replication rather than direct replication is provided as the phenotypes available to us were not identical for the Nottingham and ECLIPSE populations. Furthermore, the rarity of the G/G genotype resulted in no individuals with this genotype in either dataset used to study inflammatory cells. These findings are consistent with cigarette-induced emphysema as they provide a correlation between the suggested macrophage recruitment role of MMP-12 in mice and humans. ${ }^{3}$ It is interesting that activity differences in the protease appear to translate to COPD-specific phenotypes. It would be worthwhile determining whether the SNP has a relationship with other COPD processes such as the levels of pathological agents secreted by alveolar macrophages or increased cleavage of $\alpha_{1}$-antitrypsin, a substrate for MMP-12. ${ }^{20}$

Another finding of this study was the association between the rs652438 SNP and severity of emphysema, with A/A homozygotes having more severe emphysema. Smokers with a less active form of MMP-12 may therefore be partially protected from severe emphysema. However, despite this being a single test of association, it persisted after adjusting for confounding factors $(p=0.016)$. Thus, although these associations are consistent, further validation is required through replication in a larger cohort for macrophage infiltration and emphysema.

It has been known for some time that only a proportion of individuals who smoke develop $\mathrm{COPD},{ }^{21}$ and our findings contribute some genetic and functional evidence to the concept of the 'susceptible smoker'. However, the rarity of the A/G and G/G genotypes at MMP-12 rs652438 (around 3.8\% of individuals with severe or very COPD possess at least one $\mathrm{G}$ allele ${ }^{7}$ ) suggests that a number of other loci are likely to contribute to COPD susceptibility. It should also be noted that the ECLIPSE populations used in this study were smokers with COPD and thus would be predicted to have a lower frequency of the minor $G$ allele compared with a 'healthy' smoker group. ${ }^{7}$ The rarity of these minor homozygotes suggests that, although it confers a protective effect against emphysema, it is negatively selected against. This could be due to the central role of MMP-12 in inflammatory processes and a possible protective role in cancer metastasis. ${ }^{22}$ Low MMP-12 activity in healthy individuals could thus be undesirable, resulting in susceptibility to various conditions.

MMP-12 has been implicated in a number of other diseasesspecifically, the rs652438 SNP has been implicated in lung cancer metastasis and survival ${ }^{23} 24$-and our findings may also have implications for these diseases.

Perhaps the most important finding of this study is that it provides evidence that the findings in the MMP-12 knockout mouse model are relevant to the pathogenesis of human COPD. Knockout mice have impaired levels of tumour necrosis factor $\alpha(\mathrm{TNF} \alpha)$ release in response to acute smoke exposure. ${ }^{25}$ This is interesting as TNF $\alpha$ receptor knockout mice develop far less emphysema than control mice in response to smoke exposure. ${ }^{26}$ MMP-12 has also been shown to be able to cleave $\boldsymbol{\alpha}_{1}$-antitrypsin. ${ }^{27}$ Furthermore, investigation of MMP-12 and associated pathways in COPD could lead to an intervention strategy appropriate to individuals with emphysema.

In conclusion, this study provides evidence that an exonic SNP can alter MMP-12 activity and this relates to certain aspects of the COPD phenotype. Together with previous research, this strengthens the case for the role of MMP-12 as a key protease in COPD. It is unusual to obtain correlations of genotype with phenotypic expression to the extent demonstrated in this study.

Acknowledgements The authors would like to thank the members of the Evaluation of COPD Longitudinally to Identify Predictive Surrogate End-points (ECLIPSE) Steering Committee for providing data from this cohort. We are grateful to Lorna Magowan for technical assistance.

Funding This study is funded by a Medical Research Council Capacity Building Studentship.

\section{Competing interests None.}

Ethics approval This study was conducted with the approval of the Nottingham Research Ethics Committee and all patients gave informed consent. Investigations into ECLIPSE resource were approved by the ECLIPSE steering committee. Informed consent and collection of the cohort was as described by Vestbo et al. ${ }^{11}$

Provenance and peer review Not commissioned; externally peer reviewed.

\section{REFERENCES}

1. Laurell CB, Eriksson S. The electrophoretic $\boldsymbol{\alpha}_{1}$-globulin pattern of serum in $\alpha_{1}$-antitrypsin deficiency. Scand J Clin Lab Invest 1963;15:132-40.

2. Hunninghake GM, Cho MH, Tesfaigzi $Y$, et al. MMP12, lung function, and COPD in high-risk populations. N Engl J Med 2009;361:2599-608

3. Hautamaki RD, Kobayashi DK, Senior RM, et al. Requirement for macrophage elastase for cigarette smoke-induced emphysema in mice. Science 1997;277:2002-4.

4. Molet S, Belleguic $\mathrm{C}$, Lena $\mathrm{H}$, et al. Increase in macrophage elastase (MMP-12) in lungs from patients with chronic obstructive pulmonary disease. Inflamm Res 2005:54:31-6.

5. Demedts IK, Morel-Montero A, Lebecque S, et al. Elevated MMP-12 protein levels in induced sputum from patients with COPD. Thorax 2006;61:196-201.

6. Babusyte A, Stravinskaite K, Jeroch J, et al. Patterns of airway inflammation and MMP-12 expression in smokers and ex-smokers with COPD. Respir Res 2007;8:81 
7. Haq I, Chappell S, Johnson S, et al. Association of MMP-12 polymorphisms with severe and very severe COPD: a case control study of MMPs 1, 9 and 12 in a European population. BMC Med Genet 2010;11:7.

8. Jormsjo S, Ye S, Moritz J, et al. Allele-specific regulation of matrix metalloproteinase-12 gene activity is associated with coronary artery luminal dimensions in diabetic patients with manifest coronary artery disease. Circ Res 2000;86:998-1003.

9. Belvisi MG, Bottomley KM. The role of matrix metalloproteinases (MMPs) in the pathophysiology of chronic obstructive pulmonary disease (COPD): a therapeutic role for inhibitors of MMPs? Inflamm Res 2003;52:95-100.

10. Lowrey GE, Henderson N, Blakey JD, et al. MMP-9 protein level does not reflect overall MMP activity in the airways of patients with COPD. Respir Med 2008;102:845-51

11. Vestbo J, Anderson W, Coxson HO, et al. Evaluation of COPD Longitudinally to Identify Predictive Surrogate End-points (ECLIPSE). Eur Respir J 2008:31:869-73.

12. Henderson N, Markwick LJ, Elshaw SR, et al. Collagen I and thrombin activate MMP-2 by MMP-14-dependent and -independent pathways: implications for airway smooth muscle migration. Am J Physiol Lung Cell Mol Physiol 2007;292:L1030-8.

13. Ceresa CC, Knox AJ, Johnson SR. Use of a three-dimensional cell culture model to study airway smooth muscle-mast cell interactions in airway remodeling. $\mathrm{Am} \mathrm{J}$ Physiol Lung Cell Mol Physiol 2009;296:L1059-66.

14. Bradford MM. A rapid and sensitive method for the quantitation of microgram quantities of protein utilizing the principle of protein-dye binding. Anal Biochem 1976;72:248-54

15. Shipley JM, Wesselschmidt RL, Kobayashi DK, et al. Metalloelastase is required for macrophage-mediated proteolysis and matrix invasion in mice. Proc Natl Acad Sci U S A 1996;93:3942-6.

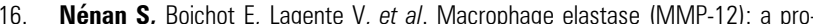
inflammatory mediator? Mem Inst Oswaldo Cruz 2005;100:167-72.
17. International HapMap Consortium. The International HapMap Project. Nature 2003;426:789-96.

18. Shapiro SD, Kobayashi DK, Ley TJ. Cloning and characterization of a unique elastolytic metalloproteinase produced by human alveolar macrophages. J Biol Chem 1993;268:23824-9

19. Bertini I, Calderone V, Fragai $\mathrm{M}$, et al. Evidence of reciprocal reorientation of the catalytic and hemopexin-like domains of full-length MMP-12. J Am Chem Soc 2008;130:7011-21

20. Banda MJ, Clark EJ, Werb Z. Limited proteolysis by macrophage elastase inactivates human alpha 1-proteinase inhibitor. J Exp Med 1980;152:1563-70.

21. Fletcher $\mathbf{C}$, Peto R. The natural history of chronic airflow obstruction. BMJ 1977;1:1645-8.

22. Houghton AM, Grisolano JL, Baumann ML, et al. Macrophage elastase (matrix metalloproteinase-12) suppresses growth of lung metastases. Cancer Res 2006:66:6149-55.

23. Su L, Zhou W, Asomaning K, et al. Genotypes and haplotypes of matrix metalloproteinase 1, 3 and 12 genes and the risk of lung cancer. Carcinogenesis 2006;27:1024-9.

24. Heist RS, Marshall AL, Liu G, et al. Matrix metalloproteinase polymorphisms and survival in stage I non-small cell lung cancer. Clin Cancer Res 2006:12:5448-53.

25. Churg A, Wang RD, Tai $\mathrm{H}$, et al. Macrophage metalloelastase mediates acute cigarette smoke-induced inflammation via tumor necrosis factor-alpha release. Am J Respir Crit Care Med 2003;167:1083-9.

26. Churg A, Wang RD, Tai H, et al. Tumor necrosis factor-alpha drives $70 \%$ of cigarette smoke-induced emphysema in the mouse. Am J Respir Crit Care Med 2004:170:492-8.

27. Gronski TJ Jr, Martin RL, Kobayashi DK, et al. Hydrolysis of a broad spectrum of extracellular matrix proteins by human macrophage elastase. J Biol Chem 1997:272:12189-94.

\section{Journal club}

\section{A new tuberculosis vaccine}

The BCG vaccine, designed as a prophylactic vaccine for pre-infection administration, is currently the only tuberculosis vaccine approved for human use.

In this study researchers developed a new multistage tuberculosis vaccine (H56) which can be used before and after exposure, and tested it in a mouse model. To construct the H56 vaccine, the authors purified the recombinant fusion protein (Ag85B-ESAT6-Rv2660c) from Escherichia coli. They hypothesised that it is possible to selectively target Mycobacterium tuberculosis (Mtb) in the persistent stage of infection by combining early protective antigens such as Ag85B and ESAT-6 (the H1 vaccine) with the latency protein Rv2660c which is involved in stress responses and characterises long-term Mtb adaptation in the immune host.

The authors assessed the effectiveness of H56, H1 and BCG vaccines administrated in mice 6 weeks before Mtb exposure. They demonstrated a statistically significant reduction in bacterial load and induction of immune response with the H56 vaccination compared to the $\mathrm{H} 1$ and BCG vaccines starting from 12 weeks after the introduction of infection. The H56 vaccine also enhanced the immunological reaction when administrated to mice with earlier treated tuberculosis infection as well as improving response in previously BCG-vaccinated mice later exposed to $\mathrm{Mtb}$

The new vaccine induces vaccine-specific polyfunctional $\mathrm{CD} 4^{+} \mathrm{T}$ cells providing efficient containment of early- and late-stage infection in addition to protection against disease reactivation. This emergence of a novel tuberculosis vaccine in mouse models is an important step forward to guide studies in humans.

Aagaard C, Hoang T, Dietrich J, et al. A multistage tuberculosis vaccine that confers efficient protection before and after exposure. Nat Med 2011;17:189-94.

\section{Wilczynska}

Correspondence to M Wilczynska, ST4 Respiratory Medicine, Glan Clwyd Hospital, Rhyl, Denbighshire LL18 5UJ, UK; mwilczynska@doctors.org.uk

Published Online First 30 March 2011

Thorax 2011;66:976. doi:10.1136/thoraxjnl-2011-200113 